\title{
Polyaniline coated on tapered multimode fiber for ammonia sensing
}

\begin{abstract}
This paper presents optical response of polyaniline coated on tapered multimode fiber towards ammonia gas. Polyaniline was deposited onto tapered multimode fiber by spraycoating method. Surface morphology of the coating was observed under scanning electron microscope. Absorbance measurement was done using spectrometer while the coated fiber was exposed to ammonia gas with concentration varies from $0.125 \%$ to $1 \%$ at room temperature. The absorbance is proportional to the ammonia concentration. The response and recovery time is 2.27 minutes and 10 minutes, respectively.
\end{abstract}

Keyword: Absorbance; Ammonia sensor; Conducting polymer; Fiber sensor; Tapered fiber 